\title{
Vetores das Leishmanioses no Parque Estadual do Morro do Diabo (PEMD), Município de Teodoro Sampaio - SP, Brasil
}

\author{
Leishmaniasis Vectors in Morro do Diabo State Park (PEMD), Teodoro Sampaio - \\ $S P$, Brazil
}

\section{Vectores de las Leishmaniasis en el Parque Estadual del Morro do Diabo, Município de Teodoro Sampaio - SP, Brasil}

\author{
Baltazar Casagrande ${ }^{1}$ \\ Raul Borges Guimarães ${ }^{2}$
}

\begin{abstract}
RESUMO: O objetivo deste trabalho foi avaliar a similaridade, a dominância e a diversidade das comunidades de flebotomíneos do interior e da borda do Parque Estadual do Morro do Diabo (PEMD). Este trabalho foi realizado por meio de coletas noturnas de flebotomíneos, utilizando armadilhas tipo Shannon e CDC. Os dados foram analisados pelo software PAST e DivEs. Foram encontrados no interior do PEMD quatro espécies diferentes de flebotomíneos, sendo 190 exemplares de Nyssomyia neivai, 128 de Pintomyia pessoai, quatro Evandromyia carmelinoi e dois Nissomyia whitmani. Já na borda foram encontradas cinco espécies diferentes, sendo 1620 exemplares de Nyssomyia neiva, 12 de Nissomyia whitmani, quatro de Evandromyia lenti, um de Psathyromyia aragaoi e um exemplar de Brumptomyia brumpti. Diante destes dados, constatou-se que o índice de similaridade entre as comunidades de flebotomíneos do interior e da borda do parque foi de 0,44,o índice de dominância de 0,50 e a diversidade com diferença significativa de 0,015. Os parâmetros obtidos e calculados foram importantes entender a similaridade e dominância entre as comunidades de flebotomíneos do parque, sendo a similaridade entre as diferentes comunidades baixa e não apresentada uma dominância de espécie. Foi encontrada uma diferença significativa na diversidade de espécies entre as amostras.
\end{abstract}

PALAVRAS-CHAVE: Leishmanioses. Geografia da Saúde. Flebotomíneos. Biogeografia.

ABSTRACT: The aim of this study was to evaluate the similarity, dominance and diversity of phlebotomine communities in the interior and edges of the Morro do Diabo State Park (PEMD). This work was carried out through nocturnal collections of sand flies, using Shannon and CDC traps. The data were analysed by PAST and DivEs software. Four different species of sand flies were found

\footnotetext{
1 Universidade Estadual Paulista (UNESP). Campus de Presidente Prudente. Faculdade de Ciências e Tecnologia (FCT), Laboratório de Biogeografia e Geografia da Saúde, Departamento de Geografia, FCT/UNESP e Professor SEDUC/MT, Escola Estadual Cleufa Hubner. Rua Roberto Simonsen, 305, Centro Educacional, CEP: 19060-900 - Presidente Prudente, SP. professor.baltazar17@gmail.com.

2 Universidade Estadual Paulista (UNESP). Campus de Presidente Prudente. Faculdade de Ciências e Tecnologia (FCT), Laboratório de Biogeografia e Geografia da Saúde, Departamento de Geografia, FCT/UNESP, Rua Roberto Simonsen, 305, Centro Educacional, CEP: 19060-900 - Presidente Prudente, SP. raul@fct.unesp.br.
} 
inside the PEMD, with 190 specimens of Nyssomyia neivai, 128 of Pintomyia pessoai, 04 Evandromyia carmelinoi and 02 Nissomyia whitmani. At the edge of the park, five different species were found, including 1620 specimens of Nyssomyia neiva, 12 of Nissomyia whitmani, 04 of Evandromyia lenti, 01 of Psathyromyia aragaoi and 01 of Brumptomyia brumpti. Based on these data, the index of similarity between sandfly communities in the interior and edge of the park was 0.44, the dominance index was 0.50 and the diversity, with a significant difference, was 0.015 . The obtained and calculated parameters were important to understand the similarity and dominance among the phlebotomine communities of the park, highlighting the low similarity between the different communities and revealing no species dominance. A significant difference in species diversity was found between samples.

KEY WORDS: Leishmaniasis. Health Geography. Sandflies. Biogeography.

RESUMEN: El objetivo de este trabajo fue evaluar la semejanza, la dominación y la diversidad de las comunidades de flebotomíneos del interior y del borde del Parque Estadual del Morro do Diabo (PEMD). Este trabajo fue realizado por medio de colectas nocturnas de flebotomíneos, utilizando trampas tipo Shannon y CDC. Los datos fueron analizados por el software PAST y DivEs. Se encontraron en el interior del PEMD cuatro especies diferentes de flebotomíneos, siendo 190 ejemplares de Nyssomyia neivai, 128 de Pintomyia pessoai, cuatro Evandromyia carmelinoi y dos Nissomyia whitmani. En el borde se encontraron cinco especies diferentes, siendo 1620 ejemplares de Nyssomyia neiva, 12 de Nissomyia whitmani, cuatro de Evandromyia lenti, uno de Psathyromyia aragaoi y un ejemplar de Brumptomyia brumpti. Ante estos datos, se constató que el índice de similitud entre las comunidades de flebotomíneos del interior y del borde del parque fue de 0,44, el índice de dominancia de 0,50 y la diversidad con diferencia significativa de 0,015. Los parámetros obtenidos y calculados fueron importantes entender la similitud y dominación entre las comunidades de flebotomíneos del parque, siendo la similitud entre las diferentes comunidades bajas y no presentando una dominación de especie. Se encontró una diferencia significativa en la diversidad de especies entre las muestras.

PALABRAS-CLAVE: Leishmaniasis. Geografía de la Salud. Flebotomíneos. Biogeografía.

\section{INTRODUÇÃO}

No campo da Geografia, muitos estudos têm sido elaborados para entender a dinâmica dos flebotomíneos e das leishmanioses devido à transformação do espaço. Fonseca (2013) selecionou 94 artigos publicados em periódicos nacionais e internacionais, de reconhecido valor na comunidade científica, que relacionam geografia e as leishmanioses. Dentre os trabalhos encontrados, $45,7 \%$ tem enfoque no estudo de vetores do agente patogênico causador das leishmanioses.

Estes vetores são pequenos insetos, denominados flebotomíneos, pertencentes à ordem Diptera, família Psychodidae, subfamília Phlebotominae. São insetos pilosos de coloração clara, facilmente identificada por apresentarem as asas sempre em pé e entreabertas quando pousados em uma superfície. Por isso, são conhecidos popularmente como cangalha, mosquito palha, birigui, tatuíra ou, ainda, como sandfly (BRASIL, 1997; MARZOCHI; SCHUBACH; MARZOCHI, 2001). 
As fêmeas de flebotomíneos são hematófagas obrigatórias. Quando realizam a hematofagia em animais silvestres, sinantrópicos ou domésticos infectados infectam-se e transmitem as Leishmanias (REY, 2011). Segundo Young e Duncan (1994), estes insetos nas Américas pertencem a apenas um gênero, Lutzomyia, enquanto Galati (2003) os inclui em vários gêneros, com destaque para: Lutzomyia, Migonemyia, Pintomyia, Bichronomyia, Psychodopygus, Nyssomyia etrichophoromyia.

A leishmaniose pode manifestar-se clinicamente em três formas: a cutânea, a cutaneomucosa e a visceral. As formas cutânea e cutaneomucosa são chamadas de Leishmaniose Tegumentar Americana (LTA), enquanto a forma visceral é a denominada Leishmaniose Visceral Americana (LVA).

A LTA encontra-se entre as seis doenças infecto-parasitárias de maior importância e representa um dos grandes problemas de saúde pública nas Américas. Distribui-se amplamente, estendendo-se do sul dos Estados Unidos até o norte da Argentina. No Brasil, tem sido notificada em praticamente todos os estados. Na década de 1950 houve uma diminuição geral da ocorrência da Leishmaniose Tegumentar Americana (BRASIL, 2007).

A LVA é uma doença endêmica, no entanto têm sido registrados surtos frequentes. Inicialmente, sua ocorrência estava limitada a áreas rurais e a pequenas localidades urbanas. Todavia, hoje, encontra-se em franca expansão para grandes centros. A LVA está distribuída em 21 unidades da federação, atingindo as cinco regiões brasileiras. Nos últimos dez anos a média anual de casos de LVA foi de 3.379 casos e a incidência de 1,9 casos por 100.000 habitantes. Os dados epidemiológicos dos últimos 10 anos revelam a Peri urbanização e a urbanização da LVA, destacando-se os surtos ocorridos no Rio de Janeiro (RJ), Belo Horizonte (MG), Araçatuba (SP), Santarém (PA), Corumbá (MS), Teresina (PI), Natal (RN), São Luís (MA), Fortaleza (CE), Camaçari (BA) e, mais recentemente, as epidemias ocorridas nos municípios de Três Lagoas (MS), Campo Grande (MS) e Palmas (TO) (BRASIL, 2010).

Até os anos de 1990 não foi notificado nenhum caso de transmissão autóctone de LVA no estado de São Paulo. Contudo, alguns casos importados eventuais foram diagnosticados, devido à migração. A partir desta data, no município de Araçatuba, região noroeste do estado, foram detectados cães com suspeita de LVA e apresenta de Leishmania sp. em exame parasitológico direto. Este foco associado à presença do inseto vetor, Lutzomyia longipalpis, encontrado no município em 1997, desencadeou uma investigação epidemiológica que levou a identificação do agente causal da doença, confirmando a transmissão autóctone de LVA em cães na área urbana de Araçatuba e, posteriormente, também em outros municípios, totalizando 23 municípios na região Oeste do estado. Em 1999 houve a confirmação do primeiro caso humano de LVA autóctone do Estado de São Paulo. 
Vetores das Leishmanioses no Parque Estadual do Morro do Diabo (PEMD), Município de Teodoro...

Desta forma, o objetivo deste trabalho é avaliar a similaridade, a dominância e a diversidade das comunidades de flebotomíneos do interior e da borda do Parque Estadual do Morro do Diabo (PEMD).

\section{CARACTERIZAÇÃO GEOGRÁFICA DA ÁREA EM ESTUDO}

Na região do Pontal do Paranapanema, especificamente no município de Teodoro Sampaio, o processo de antropização está ocorrendo principalmente com a expansão da atividade canavieira. As paisagens estão sofrendo profundas transformações com o forte processo migratório, a ocupação da área urbana e os desmatamentos em áreas florestais, entre outras atividades.

Estas transformações no espaço geográfico têm gerado agravos à saúde ambiental, relacionado à perda da biodiversidade, que elimina predadores naturais dos vetores dos microrganismos causadores das doenças e cria a possibilidade de novas infestações. As modificações nos aspectos socioambientais apresentam mudanças no comportamento dos flebotomíneos, podendo sofrer o desaparecimento temporário ou permanente de algumas espécies que tem como seu habitat natural áreas florestadas, porém algumas espécies se concentram nas áreas de preservação e outras se adaptam aos ambientes antropizados (FORATTINI, 1973). Localizada a oeste do estado de São Paulo, a região do Pontal do Paranapanema encontra-se entre a confluência dos rios Paraná e Paranapanema. É uma região com forte predominância de atividade agropecuária, que a partir da década de 1950 foi transformada em extensas áreas de monoculturas e pastagem (VALLADARES-PÁDUA, 2002).

O PEMD também é localizado no Município de Teodoro Sampaio, entre as coordenadas UTM 352164mE - 376044mE e 7526622mS - 7489537mS. Criado a princípio como Reserva Estadual, foi transformado em parque por meio Decreto Estadual № 25.342 e contava com 37.156,68 hectares (figura 1). Quando foi construído o reservatório da UHE de Rosana, foram desapropriados 3.000 hectares da área original às margens do rio Paranapanema, de acordo com o Decreto Estadual № 28.169 de 21 de janeiro de 1988. Hoje o PEMD conta com 34.156,68 hectares.

Recentemente, nos anos de 2000, foi introduzida a lavoura canavieira para fins de produção de etanol. Ainda de acordo com Valladares-Pádua (2002), no ano de 1941 a região do Pontal do Paranapanema era composta por vastas áreas de florestas, perfazendo um total de 247.000 hectares. A área foi transformada pelo Decreto Estadual $n^{\circ} 12.279 / 41$ em Reserva Florestal. Contudo, no início dos anos de 1950, a área foi distribuída para agricultores por ação governamental. Isso reduziu a cobertura vegetal da região, substituída principalmente por lavouras e pastagens. Hoje se limita a áreas isoladas de floresta, estando 
sua maior parte concentrada no Parque Estadual Morro do Diabo (PEMD), com aproximadamente 34.000 hectares.

Figura 1 - Recorte empírico da pesquisa

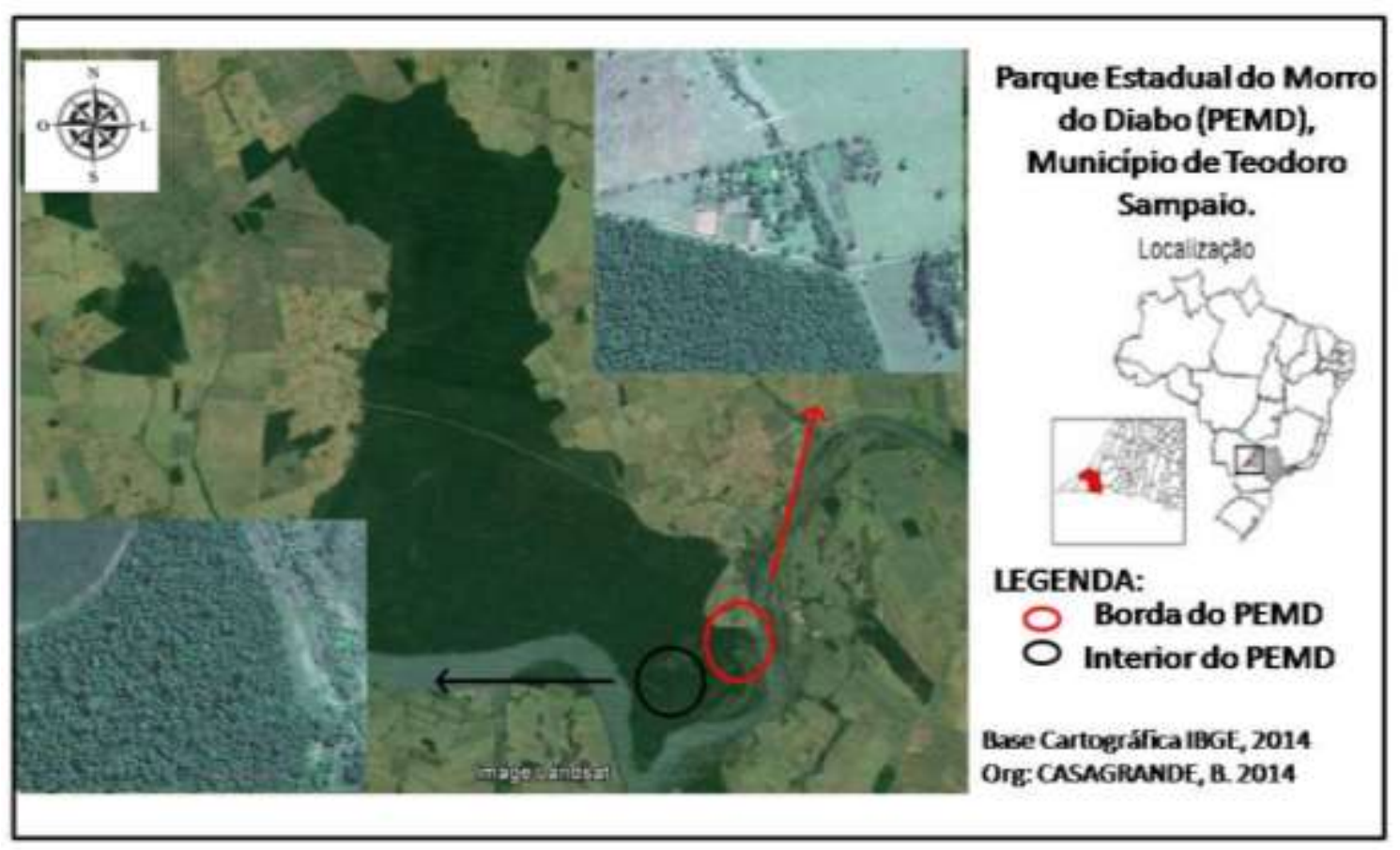

Fonte: Google Earth (2014).

Com relação às características físicas, o Pontal do Paranapanema encontra-se na província geomorfológica do Planalto Ocidental do estado de São Paulo, apresentando colinas amplas e baixa declividade, tratando-se de áreas originárias do Grupo Bauru,com solos predominantemente do tipo latossolos, com baixa fertilidade e alta permeabilidade (IPT, 1981).

De acordo com a classificação de Koppen, o clima da região é do tipo Aw, quente e úmido. Para Monteiro (1973), a região destes parques, assim como toda a área que engloba o Pontal do Paranapanema é conhecida como área de transição climática, controlada pela dinâmica de massas de ar. Estas massas de ar determinam um verão chuvoso e um inverno seco. Segundo Sparoveket, Van Lier e Dourado Neto (2007), a classificação do clima para a região é denominada clima típico de savanas, com temperaturas médias acima dos $18{ }^{\circ} \mathrm{C}$, temperaturas negativas no inverno e chuvas fortes no verão.

As florestas desta região são do tipo floresta estacional semidecidual, Mata Atlântica (TRINDADE GALO; NOVO, 1998). Trindade Galo (2000) estabeleceu uma classificação mais detalhada para o Parque Estadual do Morro do Diabo, utilizando a aplicação de redes neurais definida pela inclusão da textura de dados multiespectrais e encontrou seis tipos de classes de vegetação no parque (Floresta Primária, Floresta Secundaria Estabelecida, 
Floresta Secundaria em Regeneração, Vegetação em estágio inicial de sucessão natural, Vegetação Hidrófila e Macega).

\section{METODOLOGIA}

Este trabalho foi realizado com coletas noturnas de flebotomíneos (das 18 às 21 horas) utilizando armadilhas tipo Shannon (SHANNON, 1939) e Center of Disease Control (CDC), no interior e na borda do PEMD (SUDIA; CHAMBERLAIN, 1962).

A armadilha tipo Shannon foi alimentada com um lampião agás de 500 velas (Figura 2). Nesta, utilizou-se o tubo de sucção manual - capturador de Castro, para sugar o inseto quando o mesmo pousava sobre a armadilha (BRASIL, 1996).

Figura 2 - Armadilha tipo Shannon e capturador de Castro, respectivamente

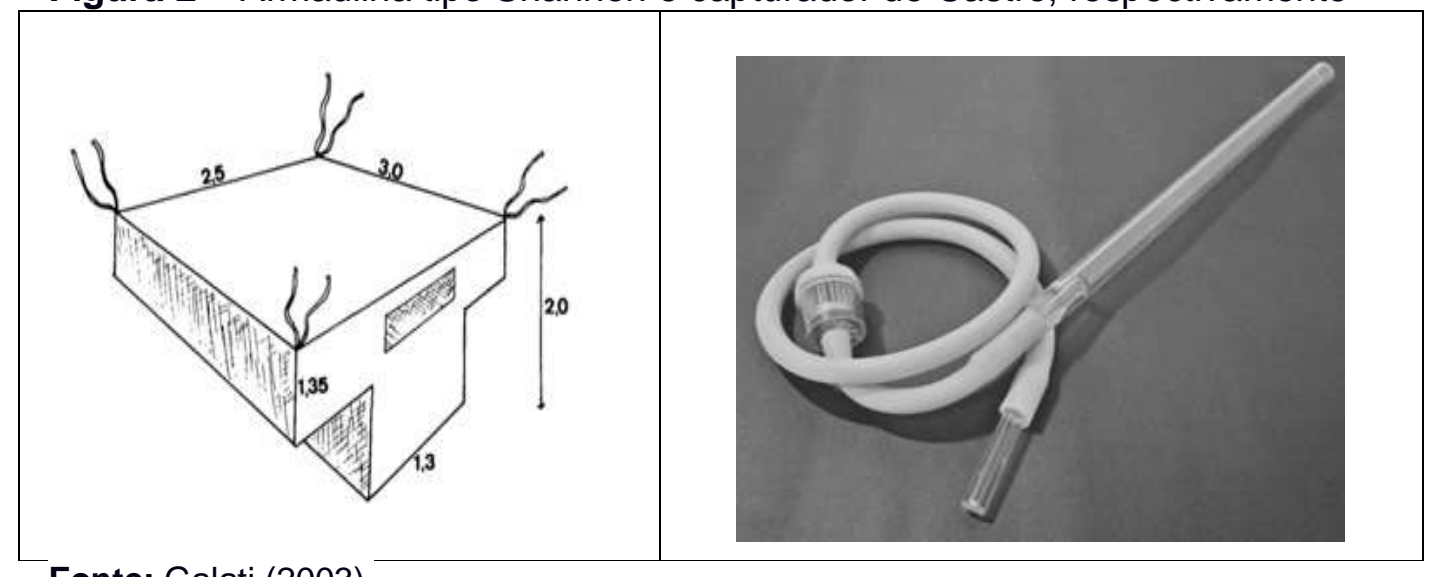

Fonte: Galati (2003).

A armadilha CDC era automática, ou seja, sugava o inseto por meio de um exaustor alimentado por uma bateria de 12 volts e 7,5 amperes, sem a manipulação do homem, e o inseto era atraído por uma lâmpada que também era alimentado pela mesma bateria (Figura $3)$.

Depois de coletados os insetos foram levados para o laboratório, onde foram sacrificados através da instilação de acetato de etila. Em seguida, submetidos à triagem em placas de Petri, observados em microscópio estereoscópico, para separar os flebotomíneos dos demais insetos. Posteriormente, os flebotomíneos triados são preparados em lâminas de microscópio para fazer a identificação quanto ao gênero e a espécie (figura 4).

Os dados foram analisados pelo software PAST 2.17 e DivEs 3.0, calculando-se a similaridade, pelo índice de Sorensen, a dominância pelo índice de Simpson e a diversidade pelo índice de Shannon-Wiener.

No software PAST 2.17 foi calculado o coeficiente de similaridade de Sorensen (Czekanovski-Dice-Sørensen), que expressa o grau no qual duas amostras são 
semelhantes para as espécies presentes nos ambientes, que se refere à alteração de espécies entre as duas amostras (MAGURRAN, 1988; BAEVe PENEV, 1995; PIELOU, 1975). Este índice pode ser obtido com base, diretamente, ou por meio de dados qualitativos ou quantitativos, métodos de gestão e classificação das comunidades (BAEV; PENEV, 1995).

Figura 3 - Armadilhas tipo CDC com as dimensões

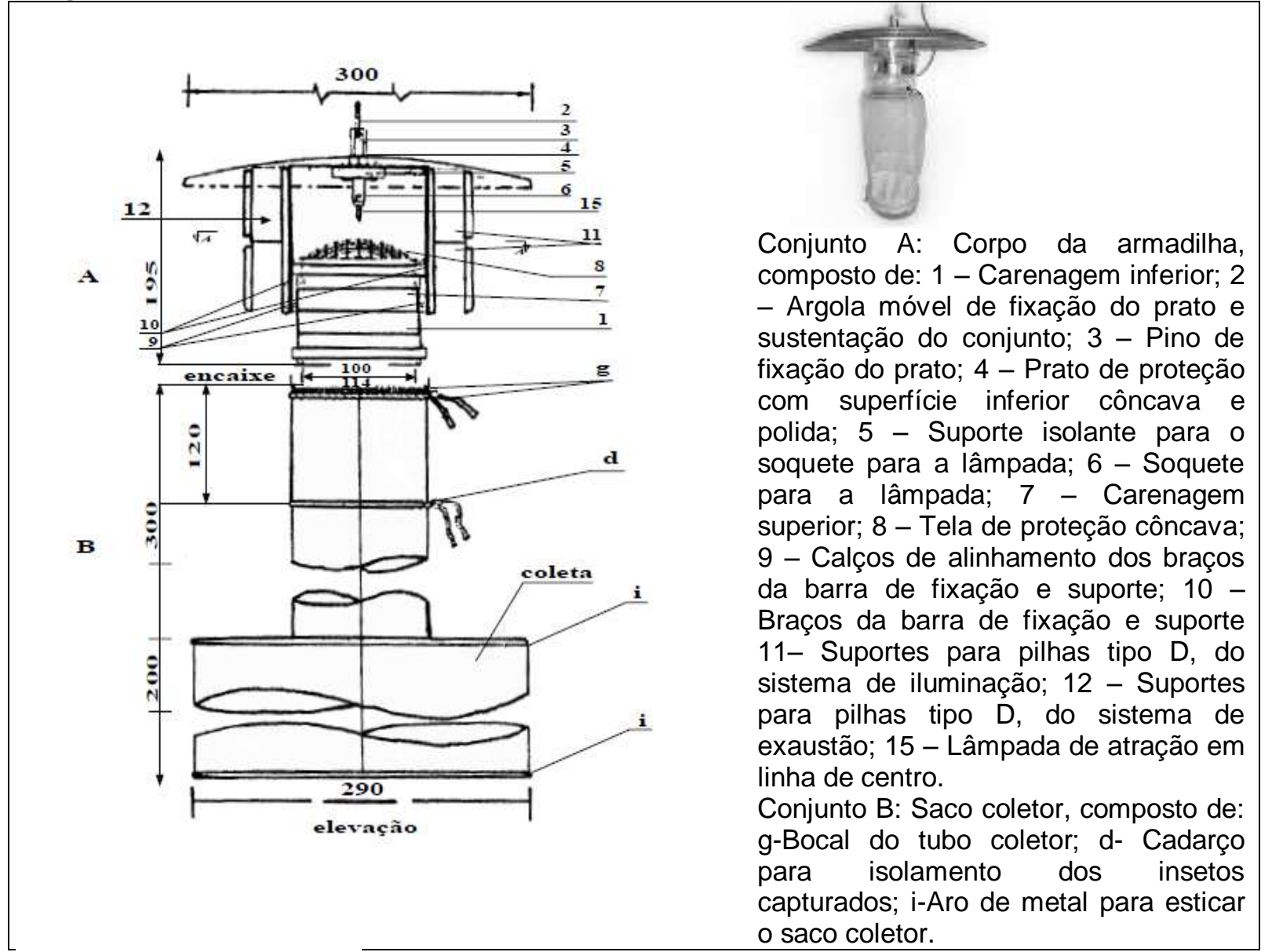

Fonte: Pugedo et al. (2005).

Desta forma, este índice relaciona o número de espécies em comum com a média aritmética das espécies em ambos os locais (MAGURRAN, 1988), conforme equação 1.

Onde,

$$
I_{s}=\frac{2 c}{a+b}(1)
$$

$a=$ número total de espécies no local $A$ (interior do PEMD)

$b=$ número total de espécies no local $B$ (borda do PEMD)

$c=$ somatório do numero de espécies nos dois locais.

Também se calculou no software PAST 2.17 a dominância pelo índice de Simpson, que manifesta a probabilidade de os indivíduos serem da mesma espécie em uma amostra, 
conforme equação 2. Está fortemente influenciado pela importância das espécies mais dominantes (MAGURRAN, 1988; PEET, 1974).

$$
\chi=\sum p i^{2}(2)
$$

Onde,

$\mathrm{p}_{\mathrm{i}}=$ número de indivíduos por espécie, dividido pelo número total de indivíduos da amostra.

Figura 4 - Preparação dos flebotomíneos para identificação

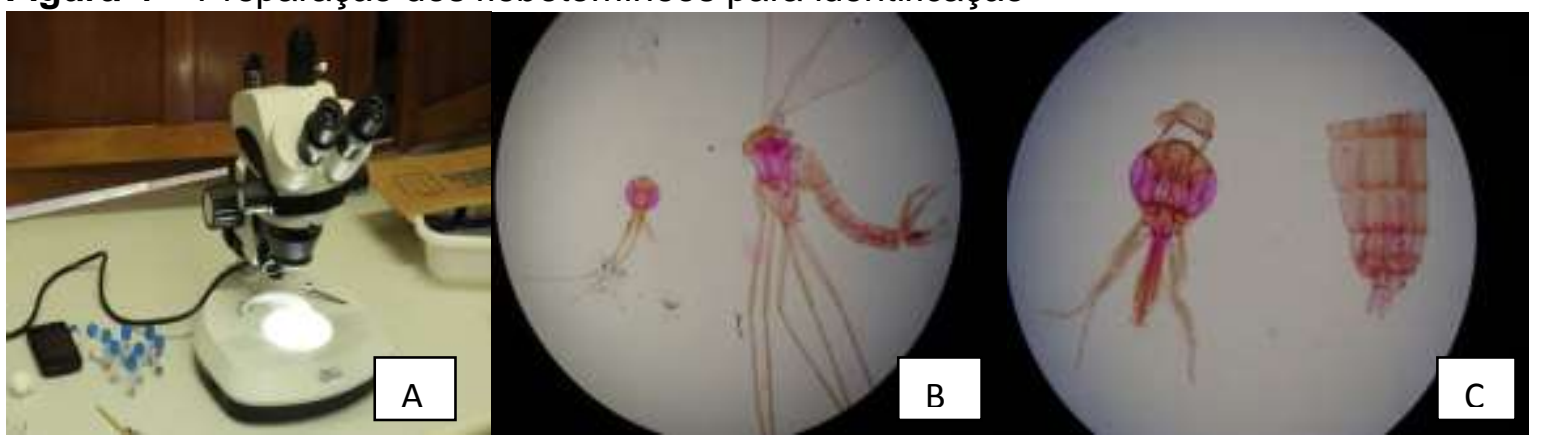

A - Microscópio estereoscópico, B - Exemplar macho e C - Exemplar fêmea. Organização: Casagrande, 2017.

No Software DivEs 3.0, calculou-se a diversidade de Shannon-Wiener.

$$
H^{\prime}=\operatorname{SUM}\left[\left(p_{i}\right) x \operatorname{In}\left(p_{i}\right)\right](3)
$$

Onde,

$S U M=$ Somatório

$p_{i}=$ proporção de amostra total representada por espécies ie numero de indivíduos de espécies i por número total de amostras

In = diversidade máxima possível.

Este índice demonstra uniformidade dos valores de importância através de todas as espécies da amostra. Mede o grau de incerteza média na previsão de que as espécies pertencem a um indivíduo escolhido aleatoriamente de uma coleção (MAGURRAN, 1988; PEET, 1974; BAEV; PENEV, 1995). O índice parte do princípio de que os indivíduos são selecionados aleatoriamente e todas as espécies estão representadas na amostra. Assume valores entre zero em que uma única espécie, e o logaritmo de $S$, quando todas as espécies são representadas pelo mesmo número de indivíduos (MAGURRAN, 1988).

\section{RESULTADOS E DISCUSSÕES}

No estado de São Paulo a transmissão da LTA é caracterizada por dois perfis epidemiológicos diferentes: um envolve aqueles indivíduos que, em contato com o ciclo enzoótico silvestre, são infectados e o outro está ligado à transmissão domiciliar, em localidades que ocorreram profundas modificações do ambiente natural, envolvendo ainda 
os animais sinantrópicos e espécies de flebotomíneos que vêm se adaptando aos ambientes rurais e periurbanos. Já o outro perfil epidemiológico, ligado à transmissão domiciliar, está estritamente vinculado à retirada da cobertura vegetal, que precedeu a construção das ferrovias. Pessoa e Pestana classificaram as regiões do estado de São Paulo como de alta e de baixa endemicidade para LTA, sendo a região do Pontal do Paranapanema classificada como alta endemicidade (ALESSI et al., 2009).

Foram encontradas no interior do PEMD quatro espécies diferentes de flebotomíneos, sendo elas Nyssomyia neivai, Pintomyia pessoai, Evandromyia carmelinoi e Nissomyia whitmani. Já na borda do PEMD foram encontradas cinco espécies diferentes de flebotomíneos: Nyssomyia neivai, Nissomyia whitmani, Evandromyia lenti, Psathyromyia aragaoi e Brumptomyia brumpti (Tabela 1 e Figura 5).

Tabela 1 - Flebotomíneos encontrados no interior e na borda do PEMD, 2013

\begin{tabular}{l|c|c|c|c|c|c|c|c|}
\hline Ponto & Ny. neivai & Pi. pessoai & Ev. camelinoi & Ev. lenti & Os. aragaoi & Ny. whitmani & Br. brumpti & \\
\hline Interior & 190 & 128 & 4 & 0 & 0 & 2 & 0 & 324 \\
\hline Borda & 1620 & 0 & 0 & 4 & 1 & 12 & 1 & 1638 \\
\hline Total & 1810 & 128 & 4 & 4 & 1 & 14 & 1 & 1962 \\
\hline
\end{tabular}

Organização: Casagrande, 2017.

Figura 5 - Flebotomíneos encontrados no interior e na borda do PEMD, 2013

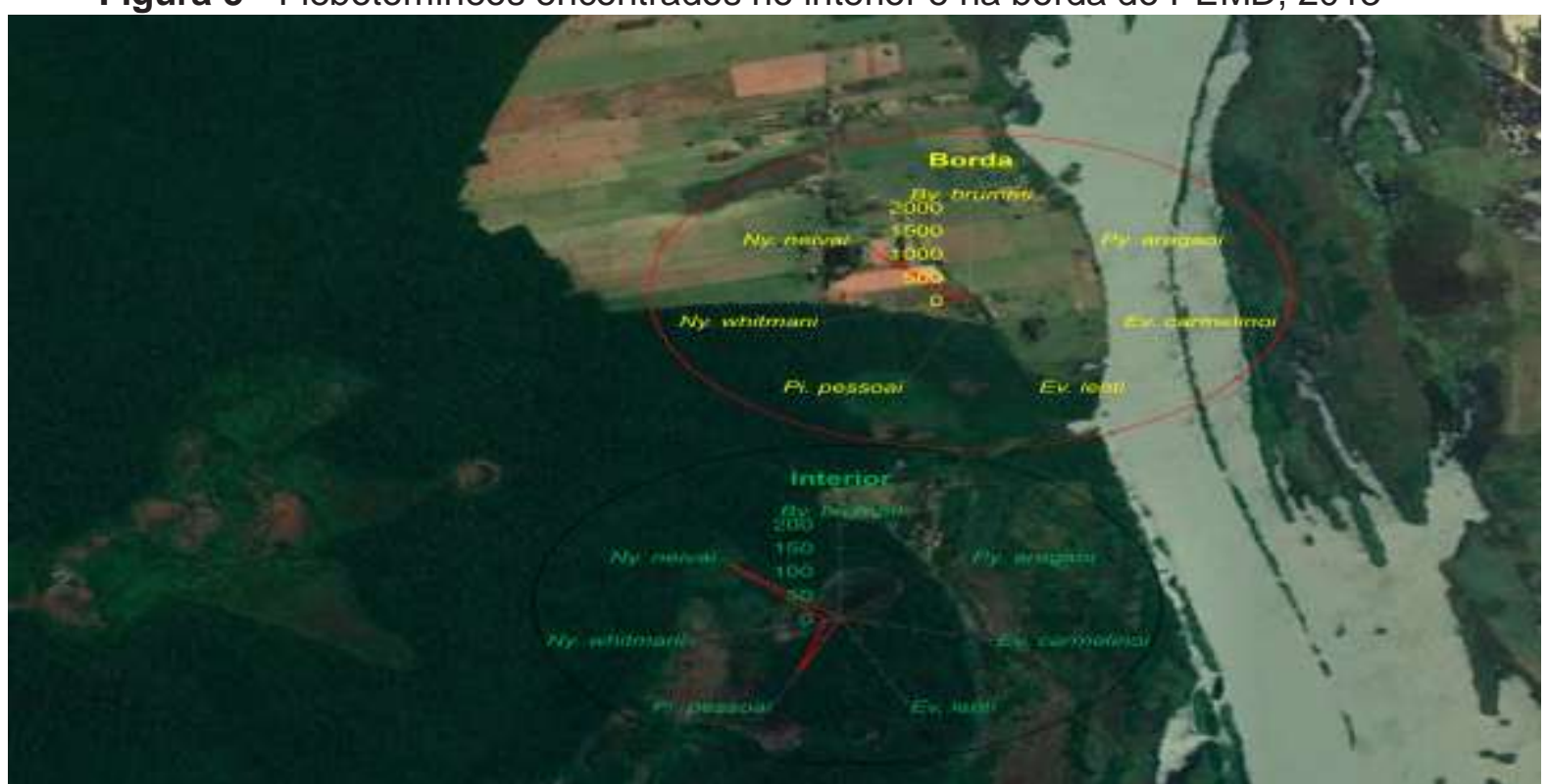

Organização: Casagrande, 2017.

Moreno (2001) relata que a perda da biodiversidade é causada, principalmente, pela ação antrópica e que isso justifica, na maioria dos casos, a criação de Áreas de Proteção Ambiental. Os dados apresentados neste trabalho demonstram que a similaridade entre as espécies do interior (preservado) com a borda (antropizada) é diferente, com um índice de 0 , 444 (tabela 2). 
Tabela 2 - Índice de similaridade entre as comunidades de flebotomíneos do interior e da borda do PEMD, 2013

\begin{tabular}{|lcc|}
\hline Ponto & Interior & Borda \\
\hline Interior & 1 & 0.44444 \\
Borda & 0.44444 & 1 \\
\hline
\end{tabular}

Organização: Casagrande, 2017.

Para os índices de similaridade e dominância, entre as comunidades de flebotomíneos do interior e da borda do Parque, o cálculo resultou em um valor de 0,44444 e 0,5, respectivamente (Tabelas 2 e 3 ).

Tabela 3 - Índice de dominância entre as comunidades de flebotomíneos do interior e da borda do PEMD, 2013

\begin{tabular}{|lcc|}
\hline Ponto & Interior & Borda \\
\hline Interior & 1 & 0.5 \\
Borda & 0.5 & 1 \\
\hline
\end{tabular}

Organização: Casagrande, 2017.

Apesar do índice de dominância apresentada ser de 0,5, ou seja, semelhança no número de espécies coletadas entre o interior e a borda do parque, existe diferenças significativas entre estes ecótopos. Esta diversidade reflete a coexistência entre organismos, que interagem através da competição no mesmo ambiente e que são influenciados por diferentes fatores. Isso implica na diferença da similaridade comentada anteriormente.

Os escritos de Huston (1995) e Silveira-Neto, Nakano e Nova (1976) são confirmados neste caso, já que estamos tratando de duas áreas com diferenças estruturais. A primeira é uma área do interior do Parque, que se encontra em estágio de conservação e a segunda é à borda do Parque, com estágio avançado de atropização. A primeira apresentou menor quantidade de indivíduos, 324 e maior equilíbrio de espécies. Já a segunda apresentou 1646 indivíduos com prevalência de uma única espécie, neste caso a Nyssomiya neivai.

A amostra 1, com 324 indivíduos, apresentou uma diversidade de Shannon-Winer de 0,3325 e a amostra 2, com 1646 indivíduos, o índice de 0,0306 (Quadros 1 e 2). O Teste t, para comparação entre duas diversidades de Shannon-Wiener, demonstrou que há uma diferença significativa entre as diversidades, a $5 \%$ de probabilidade, com valor de 0,015 (Quadro 3).

Para combinar riqueza e uniformidade, o índice de diversidade de Shannon-Wiener é o mais indicado, pois ele atribui maior peso às espécies menos encontradas. É relativamente, independente do tamanho da amostra e apresenta uma distribuição normal, contando que $\mathrm{N}$ seja um número inteiro (RODRIGUES, 2004). Com a intenção de testar se existem diferenças entre as comunidades de flebotomíneos do interior e da borda do PEMD 
calculou-se a diversidade de Shannon-Wiener e constatou-se que há diferença significativa entre as diversidades, segundo o teste t para $\mathrm{H}^{\prime}$ a $5 \%$ de probabilidade (quadros 1, 2 e 3).

Quadro 1 - Resultados da amostra 1 para o cálculo de diversidade de Shannon-Wiener

\begin{tabular}{|c|c|}
\hline \multicolumn{2}{|c|}{ Dados de: Amostra 1 (interior) } \\
\hline Número de individuos (n): & 324 \\
\hline Somatorio de fii $\times \log (i):$ & $7,056,964$ \\
\hline Somatório de fi x Log (fi) ${ }^{2}$ : & $15,566,103$ \\
\hline Variância (SH'1): & 0.0002 \\
\hline Diversidade $\left(\mathrm{H}^{\prime} 1\right)$ : & 0.3325 \\
\hline
\end{tabular}

Organização: Casagrande, 2017.

Quadro 2 - Resultados da amostra 2 para o cálculo de diversidade de Shannon-Wiener.

\begin{tabular}{|c|c|}
\hline \multicolumn{2}{|c|}{ Dados de: Amostra 2 (borda) } \\
\hline Número de individuos (n): & 1646 \\
\hline Somatbrio de fif $\times \log (\hbar)$ & $52,439,318$ \\
\hline Somatório de fi $x \log (f i)^{2}:$ & $168,077,961$ \\
\hline Variancia (S21'2): & $\mathbf{O}$ \\
\hline Diversidade $\left(\mathrm{H}^{2} \mathrm{2}\right)$ : & 0.0306 \\
\hline
\end{tabular}

Organização: Casagrande, 2017.

Quadro 3 - Resultados da Análise do Teste t da Diversidade

\begin{tabular}{|c|c|}
\hline Diferenca de SH'1 e SH'2: & 0.015 \\
\hline Valor de t: & 201,878 \\
\hline Valor de v (GL): & 464 \\
\hline$[P=0,050042]$ & - \\
\hline Valor T Tabelado t $(?=0,05)(2,464)$ & 1,965 \\
\hline \multicolumn{2}{|c|}{$\begin{array}{l}\text { Dail: Considera-se que há diferenca significativa entre } \\
\text { as diversidades, segundo o teste t para H' a } 5 \% \text { de } \\
\text { probabilidade. }\end{array}$} \\
\hline
\end{tabular}

Organização: Casagrande, 2017.

Segundo Moreno (2001), a biodiversidade em geral é regulada por vários fatores, em várias escalas. Pensando geograficamente, os fatores são bióticos e abióticos e a questão das escalas pode ser pensada a partir de uma visão de sistemas e subsistemas, isto é, uma visão geossistêmica. 
Vetores das Leishmanioses no Parque Estadual do Morro do Diabo (PEMD), Município de Teodoro...

Para Huston (1995), as espécies podem coexistir em altas quantidades nos ambientes em equilíbrio competitivo, onde a competição por fontes críticas não ocorre. Para SilveiraNeto, Nakano e Nova (1976), onde ocorrem os fatores limitantes a competição interespecífica é mais intensa, sendo assim os índices de biodiversidade são mais baixos, e aumenta o número de espécies comuns e diminui o número das mais raras.

\section{CONSIDERAÇÕES FINAIS}

As leishmanioses ainda são um problema de saúde pública, pois as mesmas continuam com altas taxas de incidência e prevalência em todo mundo. Isto se deve, principalmente, pelas modificações ambientais no espaço geográfico e a adaptação do vetor aos novos habitats de paisagens antropizadas e construídas.

Os parâmetros obtidos e calculados foram importantes para demonstrar a similaridade e dominância entre as comunidades de flebotomíneos do interior e da borda do Parque, sendo a similaridade entre as diferentes comunidades baixa, e não apresentar uma dominância de espécie.

Foi encontrada uma diferença significativa na diversidade de espécies entre as amostras. Assim, confirma-se que o processo de ocupação do espaço influencia no comportamento do vetor do agente infeccioso causador das leishmanioses, assim como no padrão da doença.

A interferência do homem na paisagem geográficapode alterar a fauna flebotomínica. Isto permite inferir que pode ser avaliado o impacto ambiental tendo por base a análise faunística de insetos como indicadores ambientais.

Nesse sentido, avaliar as comunidades de flebotomíneos, identificando as características das espécies em ambientes diferentes, pode auxiliar no controle e minimização dos casos das leishmanioses.

\section{REFERÊNCIAS}

ALESSI, C. A. C. et al. American curaneous leishmaniasis in the Pontal of Paranapanema SP, Brasil: ecological and entomological aspects. Revista do Instituro de Medicina Tropical de São Paulo, São Paulo, v. 51, n. 5, p. 272-282, jan. 2009.

BAEV, P. V.; PENEV, Y. L. D. Biodiv: program for calculating biological diversity parameters, similarity, niche overlap, and cluster analysis. Sofia-Moscow: Pensoft, 1995.

BRASIL. Controle, diagnóstico e tratamento leishmaniose visceral (calazar). Brasília: Fundação Nacional de Saúde, 1996.

Leishmaniose tegumentar americana no Brasil (Ferida Brava). Brasília: Centro Nacional de Epidemiologia, 1997. 
Manual de vigilância da leishmaniose tegumentar americana. Brasília:

Secretaria de Vigilância em Saúde, 2007.

Manual de vigilância da leishmaniose tegumentar americana. 2. ed. Brasilia:

Secretaria de Vigilância em Saúde, 2010.

FONSECA, E. D. S. Visão geográfica integrada das estratégias de controle das leishmanioses no município de Teodoro Sampaio, SP, com o apoio do geoprocessamento. 2013. 248 f. Tese (Doutorado em Geografia) - Universidade Estadual de Paulista, Presidente Prudente.

FORATTINI, O. P. Psychodidae. In: Entomologia médica. São Paulo: USP, 1973. p. 213-569.

GALATI, E. A. B. Classificação de phlebotominae- morfologia e taxonomia. In: RANGEL, E. F.; LAISON, R. Flobotomíneos do Brasil. Rio de Janeiro: Fiocruz, 2003. p. 23-51.

GOOGLE EARTH. Imagem da região do Pontal do Paranapanema: (Parque Estadual do Morro do Diabo). 2014. Disponível em:

<https://www.google.com.br/earth/download/gep/agree.html>. Acesso em: 12 jul. 2014.

HUSTON, A. H. Biological diversity: the coexistence of species on changing landscapes. Cambridge: Cambridge University, 1995.

IPT. Mapa geológico do estado de São Paulo. São Paulo: Instituto de Pesquisas Tecnológicas do Estado de São Paulo, 1981. (volumes 1 e 2).

MAGURRAN, A. E. Ecological diversity and its measurement. New Jersey: Princeton University Press, 1988.

MARZOCHI, M. C. D. A.; SCHUBACH, O. D.; MARZOCHI, B. F. Leishmaniose Tegumentar Americana. In: CIMERMAN, B. C. S. Parasitologia humana e seus fundamentos gerais. São Paulo: Atheneu, 2001. v. 1, p. 39-64.

MONTEIRO, C. A. F. A dinâmica climática e as chuvas do estado de São Paulo: estudo geográfico sob forma de atlas. São Paulo: IGEOG, v. 1, 1973.

MORENO, C. E. Métodos para medir la diversidad. Pachuca, Hidalgo: Instituto de Ecología, 2001.

PEET, R. K. The measurement of species diversity. Annual Review of Ecology and Systematics, Palo Alto, v. 5, p. 285-307, 1974.

PIELOU, E. C. Ecological diversity. New York: J. Wiley \& Sons, Inc., 1975.

PUGEDO, H. et al. HP: um modelo aprimorado de armadilha luminosa de sucção para a captura de pequenos insetos. Revista da Sociedade Brasileira de Medicina Tropical, Uberlândia, v. 38, n. 1, p. 70-72, 2005.

REY, L. Parasitologia médica. Rio de Janeiro: Guanabara Koogan, v. 1, 2011.

RODRIGUES, W. C. Homópteros (Homoptera: Sternorrhyncha) associados à tangerina cv. Poncã (Citrus reticulata Blanco) em cultivo orgânico e a interação com predadores e formigas. Seropédica: Universidade Federal Rural do Rio de Janeiro, 2004. 
SHANNON, R. C. Methods for collecting and feeding mosquitoes in jungle yellow fever studies. American Journal of Tropical Medicine, Baltimore, v. 19, n. 2, p. 131-138, 1939.

SILVEIRA-NETO, S.; NAKANO, O.; NOVA, D. B. E. N. A. V. Manual de ecologia de insetos. São Paulo: Agronômica Ceres, 1976.

SPAROVEK, G.; VAN LIER, Q. J.; DOURADO NETO, D. Computer assisted Koeppen climate classification: a case study for Brazil. International Journal of Climatology, Hoboken, v. 27, n. 2, p. 257-266, 2007.

SUDIA, W. D.; CHAMBERLAIN, R. Battery operated light trap, an improved model. Mosquito News,., v. 22, n. 1, p. 126 - 129, jan. 1962.

TRINDADE GALO, M. D. L. B. Aplicação de redes neurais artificiais e sensoriamento remoto na caracterização ambiental do Parque Estadual do Morro do Diabo. São Carlos: Universidade de São Paulo, 2000.

. ; NOVO, E. M. Índices de paisagem aplicados à análise do Parque Estadual Morro do Diabo e entorno. In: SIMPÓSIO BRASILEIRO DE SENSORIAMENTO REMOTO, 9., 1998, Santos. Anais... Santos: INPE, 1998. p. 11-18.

VALLADARES-PÁDUA, C. E. A. Módulos agroflorestais na conservação de fragmentos florestais da Mata Atlântica. Revista Experiências PDA, Brasília, v. 1, n. 1, p. 7 - 33, jan. 2002.

YOUNG, D. G.; DUNCAN, M. A. Guia para a identificação e distribuição geográfica de flebotomíneos Lutzomyia no México, Índias Ocidentais, América Central e do Sul (Diptera: Psychodidae). Gainesville: American Entomological Institute, 1994.

\section{Agradecimentos}

Agradecemos o apoio financeiro da FAPESP, ao software DivES - Diversidade de espécies, ao CETAS: Centro de Estudos do Trabalho, Ambiente e Saúde e à direção do Parque Estadual do Morro do Diabo.

Recebido: maio de 2018. Aceito: setembro de 2018. 